\title{
CARCINOME A PETITES CELLULES DU SINUS PIRIFORME : À PROPOS D'UNE OBSERVATION.
}

\author{
A. LACHKHEM, A. MARDASSI, M. ELLOUZE, D. BELHSAN, L. KOCHBATI*, S.TOUATI, S.GRITLI \\ SERVICE D'ORL ET DE CHIRURGIE CERVICO-FACIALE \\ *SERVICE DE RADIOTHÉRAPIE \\ INSTITUT SALAH AZAÏEZ DE TUNIS.
}

\begin{abstract}
RESUME
Les auteurs rapportent un cas de localisation au sinus piriforme d'un carcinome à petites cellules. Ce type histologique a été souvent décrit dans la pathologie tumorale pulmonaire. Sa localisation au sinus piriforme étant exceptionnelle. Nous présentons dans ce document les aspects endoscopiques, radiologiques, anatomopathologiques et thérapeutiques de cette tumeur qui a été diagnostiquée chez un patient de 56 ans.
\end{abstract}

Mots-clés : Sinus piriforme, carcinome à petites cellules, endoscopie, radiologie, anatomopathologie, traitement.

\begin{abstract}
The authors report a case of a small cell carcinoma of the pyriform sinus. This histological type was commonly described in the lung tumours. The pyriform sinus localization is exceptional. We present in this paper the endoscopic, radiological, pathological and therapeutic presentation of this tumor that was diagnosed in a 56 year-old man.

Keywords : Pyriform sinus, small cell carcinoma, endoscopy, radiology, anatomopathology, therapy.
\end{abstract}

\section{INTRODUCTION}

Les carcinomes à petites cellules ont été surtout décrits dans la pathologie tumorale pulmonaire. En effet, la localisation primitive extra-pulmonaire représente moins de $5 \%$ ce qui explique la rareté des publications portant sur des cas similaires. Le carcinome à petites cellule est réputé radio et chimio-sensible avec cependant une évolution locorégionale et métastatique fréquente et un faible taux de survie à 5 ans.

\section{OBSERVATIONS}

II s'agit d'un patient âgé de 56 ans, tabagique à 20 paquets/année, non alcoolique aux antécédents pathologiques d'hypertension artérielle et de diabète de type 2 . Ce patient est venu consulter pour une odynophagie isolée évoluant depuis 8 mois et s'aggravant progressivement. A l'examen, l'oropharynx était libre. Une laryngoscopie indirecte a mis en évidence une stase salivaire au niveau du sinus piriforme droit. Les aires ganglionnaires cervicales étaient libres.

Une laryngoscopie directe a été effectuée. Elle avait objectivé une formation bourgeonnante de la paroi externe du sinus piriforme droit qui a été biopsiée. La bouche de l'œsophage ainsi que le sinus piriforme gauche étaient sans anomalies. Les cordes vocales étaient d'aspect normal.

L'examen anatomopathologique avec examen immunohistochimique avait conclu à une prolifération maligne à petites cellules très indifférenciées développée au niveau du sinus piriforme droit.
Une tomodensitométrie laryngée avait objectivé une lésion hétérogène de $20 \mathrm{~mm}$ mal limitée au niveau du sinus piriforme droit, sans extension glottique ni lyse cartilagineuse (Figure 1).
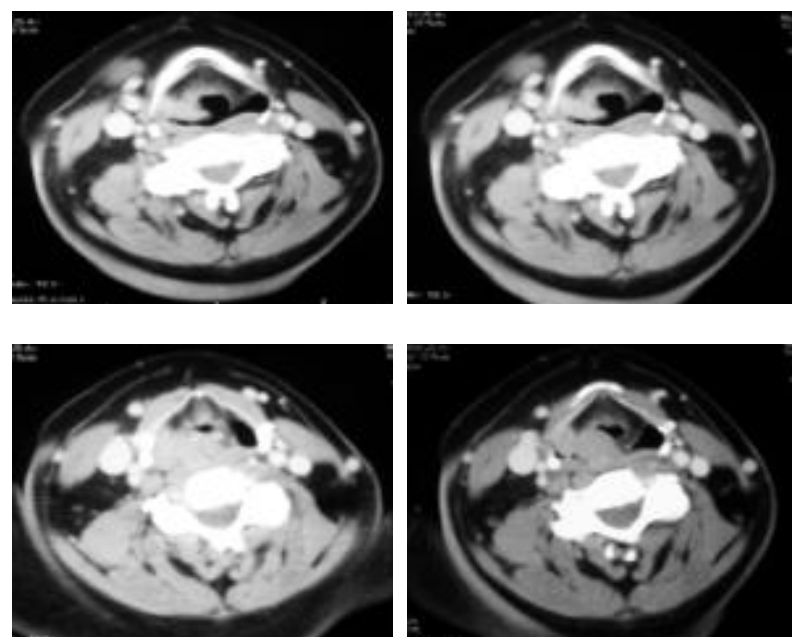

Fig. 1 : Coupes tomodensitométriques en coupes axiales et en fenêtre parenchymateuse montrant la masse bourgeonnante du sinus piriforme droit.

Le bilan fait à la recherche de localisations secondaires était négatif.

Un traitement de conservation d'organe a été décidé. Une chimiothérapie à base de 5-Fluorouracile et CDDP a été instituée mais très mal tolérée par le patient (apparition de douleurs pseudo-angineuses sans modifications élec- 
triques ni enzymatiques). Un deuxième bilan endoscopique a montré la même lésion sus-décrite du sinus piriforme droit.

Le patient a été opéré. Un curage fonctionnel droit a été effectué avec une hémi-pharyngo-laryngectomie partielle ayant permis l'exérèse de la tumeur du sinus piriforme droit. L'examen anatomopathologique définitif avait conclu après étude immuno-histochimique à un carcinome à petites cellules indifférenciées avec positivité des marqueurs cytokératine et chromogranine.

L'évolution a été bonne en post-opératoire avec diminution progressive de l'odynophagie. Après un recul actuel de 7 mois, le patient accuse des épisodes de dyspnée haute. Le bilan endoscopique de contrôle n'a pas objectivé de récidive tumorale au niveau du pharyngo-larynx.

\section{DISCUSSION}

\section{Epidémiologie :}

Les carcinomes à petites cellules ont été décrits essentiellement au niveau des poumons (1). L'origine extra-pulmonaire de ce type de tumeur ne représente que $5 \%$ des cas recensés (2). Sa localisation cervico-faciale, en général, et au niveau du pharyngo-larynx en particulier demeure très rare $(2,3,4,5)$. La majorité des patients atteints de carcinomes à petites cellules sont de sexe masculin, et comme pour la localisation pulmonaire, cette tumeur paraît intimement liée à l'intoxication tabagique $(6,7)$.

\section{Diagnostic :}

La symptomatologie qui amène les patients à consulter est généralement faite d'odynophagie, de dysphagie et parfois de dyspnée et de sensation d'accrochage alimentaire $(2,8)$. Ces plaintes justifient une laryngoscopie directe avec une biopsie et un examen anatomopathologique qui confirmeront le diagnostic. Cependant, avant de garder le caractère primitif d'un carcinome à petites cellules de l'hypopharynx, une bronchoscopie et une tomodensitométrie pulmonaire s'imposent (9).

\section{Anatomopathologie :}

Le carcinome à petites cellules représente moins de 0,5\% de toutes les tumeurs laryngées malignes (1). L'examen anatomopathologique doit être toujours couplé à l'immuno-histochimie afin d'affirmer le diagnostic. Cette étude montre que la tumeur exprime certains marqueurs neuroendocrines comme la neuron-specific enolase (NSE), la CD56, la chromogranine et la synaptophysine [8]. D'autres marqueurs peuvent aussi être exprimés par le carcinome à petites cellules comme la cytokératine, l'antigène de membrane épithélial (EMA), la Ber-ep4 et l'antigène lié à l'épithélium (ERA) (2).

\section{Traitement :}

Les procédés de chirurgie radicale dans le carcinome à petites cellules de l'hypopharynx se basant sur la pharyn- go-laryngectomie totale avec curage ganglionnaire ont échoué dans la majorité des séries de la littérature $(4,10,11,12)$. Le caractère hautement agressif de cette tumeur et le développement rapide de métastases à distance font récuser les thérapeutiques se basant sur la chirurgie seule ou la radiothérapie seule (11). En effet, les auteurs recommandent l'association chimiothérapieradiothérapie pour le traitement du carcinome à petites cellules de l'hypopharynx comme pour la localisation primitive pulmonaire $(1,3,13,14)$. Des études récentes soutiennent qu'un traitement par chimio-radiothérapie concomitante augmente l'espérance de vie $(2,9,15,16)$. Les patients présentant un carcinome à petites cellules de l'hypopharynx peu étendu, et chez qui une polychimiothérapie est contre-indiquée, sont traités par une association de chirurgie et de radiothérapie (8). Les tumeurs étendues avec des localisations secondaires à distance sont incurables et sont du ressort du traitement palliatif (8).

\section{Evolution :}

Le pronostic des carcinomes à petites cellules de l'hypopharynx est mauvais. La survie à 5 ans n'est que de $5 \%$ et la survie moyenne est de 1 an notamment dans les formes étendues $(1,17)$. Ceci est essentiellement dû à la fréquence et à la précocité des métastases à distance $(2,8)$.

\section{CONCLUSION}

Les carcinomes à petites cellules représente une tumeur rare de l'hypopharynx se caractérisant par sa haute agressivité et sa grande tendance métastatique. L'apport des marqueurs neuroendocrines est indiscutable pour le diagnostic positif. Le traitement de choix demeure l'association chimio-radiothérapie, au mieux concomitante, quoique la survie à 5 ans demeure basse n'excédant pas $5 \%$. 


\section{REFERENCES}

1) Bawa R, Wax MK. Small cell carcinoma of the tonsil. Otolaryngol Head Neck Surg 1995;113:328-33.

2) Sano M, Kitahara N, Toma M. Hypopharyngeal small cell carcinoma: A case report. Auris Nasus Larynx 2005; 32: 319-322.

3) Ferlito A, Polidoro F. Simultaneous primary oat cell carcinoma (apudoma) and squamous cell carcinoma of the hypopharynx. ORL 1980;42:146-57.

4) Ferlito A, Caruso G, Nicolai P, Recher G, Silvestri F. Primary small cell ("oat cell") carcinoma of the larynx and hypopharynx. ORL 1981;43:204-22.

5) Olofsson J, Van Nostrand AW: Anaplastic small cell carcinoma of larynx. Case report. Ann Otol Rhinol Laryngol 1972; 81: 284-287.

6) Remick SC, Hafez GR, Carbone PP: Extrapulmonary small-cell carcinoma: A review of the literature with emphasis on therapy and outcome. Medicine 1987; 66: $457-471$

7) Remick SC, Ruckdeschel JC: Extrapulmonary and pulmonary small-cell carcinoma: Tumor biology, therapy and outcome. Med Pediatr Oncol 1992 ; 20: 8999.

8) Gaba A, Mbaoma R, Breining D, Smith R, Beitler J, Haigentz M. Small-Cell Carcinoma of the Hypopharynx. Journal of Clinical Oncology 2005; 23: 20942096.

9) Galanis E, Frytak S, Lloyd RV: Extrapulmonary small cell carcinoma. Cancer 1997; 79: 1729-1736.

10) Shiotani A, Kawaura M, Fukuda H, Tanaka Y, Sako T, Sasaki S, et al. Primary small cell carcinoma of the larynx. Auris Nasus Larynx $1994 ; 21: 126-31$.
11) Ferlito $A$. Diagnosis and treatment of small cell carcinoma of the larynx: a critical review. Ann Otol Rhinol Laryngol 1986;95:590-600.

12) Ferlito A, Friedmann I. Review of neuroendocrine carcinomas of the larynx. Ann Otol Rhinol Laryngol 1989;98:780-90.

13) Sole J, Jurgens A, Musulen E, Lacasta A, Guedea F, Quer M, et al. Small cell carcinoma of the larynx: results of therapy. Bull Cancer Radiother 1994;81:45-8

14) Ferlito $A$, Barnes L, Rinaldo A, Gnepp DR, Milroy CM. A review of neuroendocrine neoplasms of the larynx: update on diagnosis and treatment. J Laryngol Otol 1998;112:827-34.

15) Noguchi K, Urade M, Sakurai K, et al: Small cell neuroendocrine carcinoma of the maxillary sinus: A case report and nude mouse transplantable model. Head Neck 2002 ; $24: 491-496$.

16) Barker JL Jr, Glisson BS, Garden AS, et al: Management of nonsinonasal neuroendocrine carcinomas of the head and neck. Cancer 2003 ; 98: 23222328.

17) Augilar 3rd EA., Robbins KT, Stephens J, Dimery IW, Batsakis JG. Primary oat cell carcinoma of the larynx. Am J Clin Oncol 1987;10:26-32. 